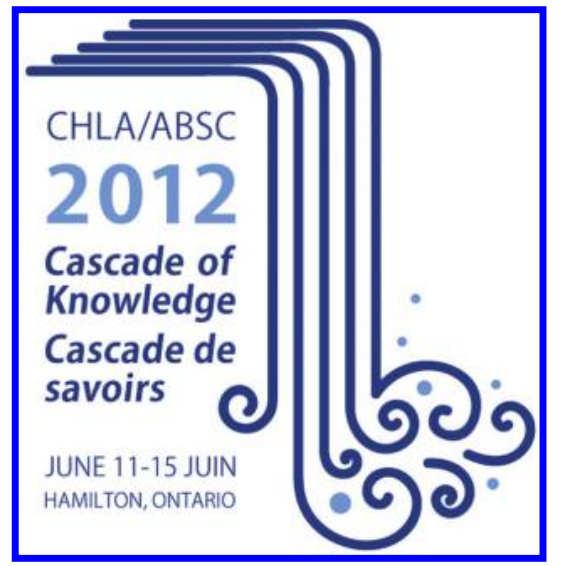

Canadian Health Libraries Association 2012 Conference

11-15 June 2012

Hamilton, Ontario, Canada
Association des bibliothèques de la santé du Canada Congrès 2012

11 au 15 juin 2012

Hamilton (Ontario) Canada

\title{
Presentation abstracts / Résumés des exposés
}





\title{
An analysis of surgical content in evidence-based resources
}

\author{
L. Banfield, N. Husain, S. Turvey, and M. Bhandari \\ Nursing, Midwifery, and Global Health Liaison Librarian, Health Sciences Library, McMaster University, \\ 1280 Main Street West, Hamilton ON, L8S 4K1
}

Introduction: There are many evidence-based resources available, particularly at the summaries level, e.g. UptoDate ${ }^{\circledR}$ and FirstConsult ${ }^{\circledR}$. However, they are broad in scope and it can be challenging to determine the extent to which individual medical specialities, such as surgery, are covered. As subscription rates continue to increase and the field of evidence-based surgery continues to expand, there is a growing need to identify resources with high levels of surgery relevant content. The purpose of this study is to develop and carry-out a method for analyzing and quantifying evidence-based summaries resources for surgery content. Methods: Based on the subscriptions available through an academic health sciences library, five summaries resources were chosen for comparison, Clinical Evidence ${ }^{\circledR}$, DyanMed ${ }^{\circledR}$, FirstConsult ${ }^{\circledR}$, PIER $^{\circledR}$, and UptoDate ${ }^{\circledR}$. Sufficient similarities in the structure and organization of these resources exist to permit the same search strategy to be used and for individual topic summaries to be retrieved. Using agreed upon criteria developed by a multidisciplinary team, the results from each resource were analyzed for relevance and categorized by surgical sub-discipline(s). Results: Preliminary results suggest that based on the evaluation criteria used, there is relatively little surgery relevant content, $8 \%-11 \%$, within these resources. Discussion: Through analyzing evidence-based summary resources from the perspective of surgery, the results of this study may be used by library staff and surgeons when selecting appropriate resources for their hospitals, clinics or for personal subscription. Further, the methods developed through this study may be used to conduct a similar analysis for other medical specialties.

\section{Googling the greys - tips for searching beyond health databases and turning that information into insights}

\author{
Sarah Bonato \\ Reference Librarian, Centre for Addiction and Mental Health, Centre for Addiction and Mental Health, \\ 33 Russell Street, Toronto, ON M5S 2S1, Canada
}

Introduction: Health information professionals are very accustom to conducting database searches, but a grey literature search in Google can really complement search results from the published literature. But although Google is great for locating sources of grey literature, it can take too much time to filter through piles of meaningless results. Geographical location could be influencing your search results immensely, because search results are becoming increasing filtered. Description: This proposed paper will demonstrate how to use the various features of Google to find quality results faster. Using
$<$ Unclesam $>$ - Google Search, Google UK, Google Canada, Google New Zealand, and Google Australia can be a great way to locate quality documents that might be otherwise missed in just a Google search. Grey literature searches for health topics will be demonstrated. Additionally, this paper will address how certain factors like your IP location and internet browser version can unknowingly influence your search results and suggest practical steps you can take neutralize this bias. Also, tips for turning grey literature search results into insights for your clients will also be discussed. Outcomes: Attendees 
will learn how to obtain higher quality results from their Google searches. Discussion: Overall this session will address the topics of identifying quality internet resources, navigating strategies and techniques, and search engine tips, and how to outsmart Google in order to find the information you need.

\title{
A portal for the Canadian Virtual Health Library I bibliothèque virtuelle Canadienne de la santé (CVHL/BVCS): accomplishments and possibilities, scope and structure - a review of best practices
}

\author{
Jim Henderson, Wendy Stark, Natalie Clairoux, Tom Flemming, Dean Giustini, and \\ Vivian Stieda \\ Librarian (Retired), McGill University 3546 Marlowe Ave., Montreal, QC H4A 3L7, 514-807-5863; Université de \\ Montréal, Hamilton ON; University of British Columbia; Health Knowledge Network / University of Calgary
}

Introduction: Coordination through CVHL/BVCS gives Canadian health libraries access to information technology they could not offer individually, thereby enhancing the library services offered to Canadian health professionals. An example is the portal being developed. Portal best practices are of increasing interest (usability.gov; Wikipedia portals; JISC subject portal project; Stanford clinical portals) but conclusive research is not yet available. This paper will identify best practices for a portal bringing together knowledge for Canadian health professionals supported through a network of libraries. Description: The portal for Canadian health professionals will include capabilities such as:

- Authentication

- Question referral

- Specialist "branch libraries"

- Integration of commercial resources, web resources and health systems data

- Cross-resource search engine
- Infrastructure to enable links from EHR and decision support systems

- Knowledge translation tools, such as highlighting of best evidence

Best practices will be determined by studying the capabilities of existing portals, including consortia/networks and individual institutions, and through a literature review. Outcomes: Best practices in portals will be reviewed. The collaboratively developed Virtual Library, currently the heart of cvhl.ca, is a unique database collecting highquality, free web documents and sites relevant to Canadian healthcare. The evident strengths of the Virtual Library will be discussed in light of best practices. Discussion: Identification of best practices will support cost-benefit analysis of options and provide direction for CVHL/BVCS. Open discussion with stakeholders (libraries and professionals) informed by this review will lead to adoption of the best technical solutions supporting Canadian health libraries and their users.

\section{Mobile device use by healthcare practitioners in Saskatchewan}

\author{
Susan Baer, Ashley Farrell, Chau Ha, Christine Neilson, Joanne Franko, \\ Dr. Michelle McCarron, and Jeff Mason \\ MLS Regina Qu'Appelle Health Region, 1440 - 14th Avenue, Regina, SK S4P OW5; Librarian, Regina Qu'Appelle \\ Health Region, Regina; Librarian, Library and Testing Services, SIAST Woodland campus, Prince Albert; SHIRP \\ Librarian, SHIRP (Saskatchewan Health Information Resources Partnership), Saskatoon; Manager, Evaluation and \\ Information Management, Saskatoon Health Region, Saskatoon; Research Scientist, Research and Health \\ Information Services, Regina Qu'Appelle Health Region, Regina; Academic Information Coordinator, Regina \\ Qu'Appelle Health Region, Regina
}


Introduction: Delivery of evidence-based information at the point-of-care can be challenging with several barriers impeding use. Mobile devices have the potential to address this challenge. Saskatchewan medical libraries are interested in learning what devices are being used and for what purposes in clinical practice. Existing literature is outdated, tends to focus on physicians, and does not cover new devices and applications. A snapshot of use/need by several professional groups would provide a more complete picture for support in Saskatchewan. Methods: A multiinstitutional, multi-disciplinary team primarily composed of Saskatchewan medical librarians selected 12 professional groups to survey across Saskatchewan. A systematic review of the literature was conducted. Research Ethics Board approval was received prior to surveys being distributed with the cooperation of the professional licensing body. A combination of paper and electronic surveys were distributed based on the licensing bodies' requirements. Results: The process of conducting the survey will be described. Frequency and descriptive statistics will be reported, along with between-groups comparisons. Discussion: Similar surveys focus on physician use of mobile devices. There are other professionals who could improve patient care by using a mobile device as well. The results of this survey will provide information on various uses and/or needs by different healthcare professionals in Saskatchewan. A separate publication of the systematic review of the literature in this area is planned for the future.

\title{
The value and impact of library and information services: the Canadian perspective
}

\author{
Joan C. Bartlett \\ Associate Professor, McGill University, School of Information Studies, 3661 Peel Street, Montreal, QC H3A 1X1
}

Introduction: In 2010, a replication of the Rochester study again highlighted the value of hospital library services and their impact on patient care. The large study population included a number of Canadian sites, although the results were aggregated along with the American data. This paper presents the findings from Canadian sites and a comparison with the American findings. Methods: Data were collected using an online survey, with questions relating to the value of library and information services, their impact on patient care outcomes, as well as the use of library and information services. A total of 1231 healthcare providers (including 371 physicians, 125 residents, and 560 nurses) from four Canadian healthcare sites responded to the survey. Follow-up qualitative interviews will supplement the survey findings. Findings: According to respon- dents the information provided by the hospital library was valuable, leading to changes in patient care $(83 \%)$, avoidance of adverse events (53\%), and improved management of the clinical situation $(71 \%)$, as well as saving time for the healthcare provider. Library resources were considered very important, ranking more highly than other resources such as colleagues or laboratory tests. The findings also identified the library resources most typically used, and their access points. Discussion: Library and information services continue to be considered valuable to healthcare providers, and have a positive impact on patient care outcomes. Implications for healthcare library and information services will be discussed, as well as the future potential and plans for a Canada-wide replication of this research.

\section{Evaluating a chat reference service in a hospital library network}

\author{
Kathryn M. E. Ranjit, Marcus Vaska, and Yongtao Lin \\ Librarian, Health Information Network Calgary Peter Lougheed Knowledge Centre, Calgary, Alberta; Health \\ Information Network Calgary, Holy Cross Site Calgary, Alberta; Alberta Health Services Cancer Care (South) \\ Calgary Alberta
}


Introduction: Recent years have seen growing demands for libraries to provide $24 / 7$ access to resources and services. Chat reference creates new and exciting opportunities for information reference services to address the challenges in using online information services, promoting the spectrum of library services to clients and identifying areas for staff competency training. Objectives: The objective of this study was to characterize how chat reference has been implemented in the Health Information Network and examine its usage by healthcare professionals since November 2009. The authors also intend to compare these services between hospital libraries and those in other academic library institutions, and identify future strategies to improve the service. Methods: A retrospective look at this service was taken from service development, implementation to one year later. Chat transcripts from November 2010-
January 2012 were analyzed using a grounded theory approach to identify the pattern of chat reference questions, and examine the usefulness and quality of service. Library staff were surveyed for feedback about how this service was implemented and how to provide further training. Results: Healthcare staff used this service for a wide variety of reasons including document delivery, literature search help, and general information about library services. Staff training in using chat for reference was important for service quality. Discussion: Chat reference has been viewed as a valuable addition to the library services in this environment. Staff has embraced this medium for providing reference services to clients. Future directions for this service will include improving reference interviewing techniques in the online medium.

\title{
An undergraduate medical school curriculum database: structure, taxonomies, and applications within the Michael G. DeGroote School of Medicine at McMaster University (Case Report)
}

\author{
Laura DiCarlo \\ Information Specialist, Division of e-Learning Innovation, Faculty of Health Science, McMaster University, \\ Michael G. DeGroote Centre for Learning, room 3515, 1200 Main Street West, Hamilton, Ontario L8N $3 Z 5$ \\ Canada
}

Purpose: To create an online curriculum database, searchable by planners and students, that describes and creates multiple points of access to undergraduate medical curriculum. Setting: The Undergraduate Medical program at the Michael G. DeGroote School of Medicine at McMaster University. The program admits 203 students each year and has three campus locations (Hamilton, Waterloo, and Niagara). The three-year program in Medicine uses a problem-based approach to learning. Method: The curriculum database has been conceptualized into three main categories: objectives, activities and assessments. Each of these three 'categories' are extensively described using a number of 'Taxonomies' or vocabularies (i.e. MeSH) and then inter-connected to create a rich relational database of curricular information. The curriculum database uses an XML and web services-based approach to allow flexibility of syndicating data to other curricular web applications, and conform to emerging standards (such as Medbiquitous Curriculum Inventory schema). Results: Users can search undergraduate medical curriculum by keyword, discipline, medical subject, time blocks, Medical Council of Canada (MCC) presentations or the Royal College of Physicians and Surgeons' CanMEDS (Canadian Medical Education Directives for Specialists) roles framework. Discussion: The curriculum database also acts to inform other applications within the school, such as the Media Catalogue and the Essential Clinical Experience (ECE) Tracker. The Media Catalogue, a repository of lectures from the undergraduate MD program, 'pulls' tags from the curriculum database related to activities in order to leverage the cataloguing already occurring in the Curriculum Database. The ECE Tracker, an assessment tool used by medical clerks to track clinical encounters, uses web services to 'pull' clinical experiences from the curriculum database, to form the core list of presentations students must experience to complete their clerkships. Future directions include the creation of a fourth 'category' within the database that will track' resources' and link these assets to activities and objectives. 


\title{
The health librarian and the iPad: mobile devices and their use in professional practice
}

\author{
Susan Murphy, Vicky Duncan, Maha Kumaran, and Mê-Linh Lê \\ MLIS, Health Sciences Library, B205.3, University of Saskatchewan, 107 Wiggins Road Saskatoon, \\ SK S7N 5E5, Canada
}

Introduction: While the literature and anecdotal experience indicate that mobile devices are being heavily adopted by health sciences faculty, students, and practitioners, there is little evidence of how this technology is being used by health librarians in their professional practice. In January 2011, health sciences librarians at the University of Saskatchewan embarked on a project to familiarize themselves with iPads and identify best practices for using them as professional tools. Methods: Over a period of eight months, eight health sciences librarians with different liaison and administrative roles journalled their experiences using the iPad, and documented information such as usage type (e.g., reference questions, meetings, conference attendance), recommended apps, technical issues, recommended readings, and general comments. In addition, monthly meetings were held to share knowledge and expertise. Summative reports submitted at the end of the project revealed a wide spectrum of experiences and opinions. Results: Numerous factors were found to impact the frequency of iPad use and satisfaction with it as a tool to aid in professional practice, including personal preferences (e.g., paper vs. electronic), past experience with Apple products, specific job function, and more. Discussion: A core suite of iPad apps relevant to academic health sciences librarians and their professional practice was identified and shared amongst colleagues at the University Library. This project highlighted the benefits and limitations of using iPads as a professional tool for academic health sciences librarians and will help other librarians determine if the purchase of iPads is worthwhile.

\section{Best practices for very large team teaching: a case study of a graduate course in health librarianship}

\author{
Dale Storie \\ John W. Scott Health Sciences Library, 2K3.28 Walter C. Mackenzie Health Sciences Centre, University of Alberta, \\ Edmonton, Alberta T6G $2 R 7$
}

Introduction: Librarians at a large academic health sciences library developed and taught the graduate level "Introduction to Health Sciences Librarianship" course at the university's School of Library and Information Studies. A research project was undertaken to assess the perceived effectiveness of very large team teaching. Description: Eleven librarians participated in the development of a new curriculum, including assignments. Each librarian taught part or all of at least one class, several helped grade assignments, and several more mentored students for their systematic review searching assignment. This active involvement in several stages differentiates the course from others that are organized by one instructor but taught primarily by guest lecturers. To evaluate the effectiveness of this team approach, evaluations were distributed to students at the mid-point of the course and again upon its completion. The librarians involved were also surveyed about their contributions and their feelings of integration with the team. Outcomes: Results show that the students are very happy with the large team teaching approach and find benefit in being exposed to the expertise of a large number of practicing professionals. The instructors valued high levels of communication among themselves and recognized the importance of consistent communications with the students. Discussion: The experience and the survey results were used to establish best practices for large team teaching, including using consensus building to establish consistency in expectations and grading, maintain effective communication among instructors, and ensure course content follows a logical order.

Other authors: Sandy Campbell; Trish Chatterley; Marlene Dorgan; Linda Seale; Linda Slater; Lisa Tjosvold; Liza Chan; Liz Dennett; Dagmara Chojecki; Thane Chambers 


\title{
A new approach to client outreach: the RQHR Library's experience with creating a webinar series
}

\author{
Caitlin Carter \\ Regina Qu'Appelle Health Region Library, 1440-14th Avenue, Regina, SK S4P OW5
}

Introduction: In the fall of 2011, work began on a series of webinars for the RQHR Library's clientele. There were several reasons to embark on this project: "Telehealth" was no longer an effective means of delivering library training; librarian burnout from repetitious training sessions; and the inability of many library users to attend the scheduled in-person training classes. The main goal is to conduct and record effective webinars to increase user comfort level with the library's website and electronic resources. Recorded webinars would be available for access 24/7. Description: Through a series of rigourously marketed live and recorded webinars created using WebEx software, patrons will learn how to effectively use the electronic resources provided by the RQHR Library. A calendar listing of the webinars will be available electronically for registration to allow users to match their schedules. The main clientele to be reached include those who are unable to attend our in-person training sessions (e.g. nurses and those on opposite shifts), as well as the rural staff. The webinar series is planned as an on-going project, as the library's website and resources change. Outcomes: Results from the webinar feedback surveys will be presented. These results will illustrate any changes in client behaviour and their thoughts on the new service. Discussion: Any challenges encountered during the project, as well as advice for those considering implementation of a similar endeavour. Future directions may include partnering with other institutions, as a way for libraries to share resources.

\section{Knowledge on the go: a survey of mobile device usage in medicine}

\author{
Jill Boruff, Dale Storie, and Dagmara Chojecki \\ Life Sciences Library, McGill University, 3655 Promenade-Sir-William-Osler, Montréal, Québec H3G 1Y6; \\ John W. Scott Library, University of Alberta, 2K3.28 Walter Mackenzie Health Sciences Centre, Edmonton, \\ Alberta T6G 2R7; John W. Scott Library, University of Alberta
}

Introduction: Little is known about medical trainees' and clinicians' current use of mobile devices for informationseeking, including the resources they use and in what context. This study was designed to better understand what types of mobile information resources this population finds valuable, how and why they find them valuable, what criteria they use in selecting resources, and what kinds of problems they encounter in using mobile resources. To investigate how students, residents and faculty members in Canadian medical faculties use mobile devices such as smart phones (e.g. iPhone, Android, Blackberry) and tablet computers (e.g. iPad), specifically in relation to their studies and professional environment. Methods: This is a multi-site study at five Canadian universities. An electronic survey was distributed by medical librarians at each university to medical students, residents, and faculty members via departmental listservs, personal contacts, and relevant websites. The survey investigates the types of information sought, frequency of and reasons for mobile device use in medical information-seeking, access barriers, support needs, familiarity with institutionally licensed resources, and personal purchasing of resources. Qualitative information was solicited by asking for a specific incident in which a mobile device was used to answer a clinical question. Participants could choose to be contacted for a follow-up interview; however, those results will not be presented here. Results: Data from over 800 survey responses, demonstrating the trends at the participating universities, will be presented. Discussion: The discussion will address how academic health libraries can effectively support mobile technology and collections in light of survey results. 
Other Authors: Jill Boruff, Dale Storie, Dagmara Chojecki, Helen Lee Robertson, Lee-Anne Ufholz, Tania Gottschalk, and Sherri Vokey.

\title{
Getting integrated: a "subversive activity" at McMaster University's Health Sciences Library
}

\author{
Elizabeth Yates \\ Special Projects, Health Sciences Library, McMaster University
}

Introduction: This case study explores instructional partnerships between liaison librarians and faculty members in the Faculty of Health Sciences at McMaster University, in Hamilton, ON. McMaster's Health Sciences Library has a notable history of liaison librarianship; as well, the novel teaching methods used in McMaster's health professional programs such as medicine, nursing, and rehabilitation sciences have fostered intriguing collaborations between faculty members and librarians. Objective: To highlight both successes and "lessons learned" and create recommendations for best practices to help information specialists/librarians build successful working relationships with their client groups. Scope: Although this study examined the environment at just one library, the results reflect and deepen the body of LIS research into faculty librarian collaboration and could potentially be generalized to a wider population. Methods: Semi-structured interviews were conducted with six liaison librarians and five faculty members between February 2011 and January 2012. Participants agreed to be named in published reports, enhancing the validity of the results. Results: McMaster's focus on evidence-based practice and problem-based learning enhance the role of health sciences librarians, who are integral components of programs including nursing, occupational therapy and physiotherapy. Integration into the medical program is less robust, reflecting a resistance to training in soft skills. Discussion: Major themes emerged showing that successful collaborations are built around acculturation; making strategic connections; and getting involved in institutional activities. Challenges include time pressures; communication missteps; and lack of "big picture" planning. Interestingly, contrary to other research findings, faculty members in this study expressed respect and admiration for their librarian colleagues.

\section{Library services for medical students, clerks, and residents at a hospital site: needs assessment and negotiation}

\author{
Brooke Ballantyne Scott and Allison Lambert \\ Library, Royal Columbian Hospital, 330 East Columbia Street, New Westminster, BC; Royal Columbian Hospital, \\ 330 East Columbia Street, New Westminster, BC
}

Introduction: Medical library services are often made available to medical students, clerks and residents while they are on rotation or placement at a hospital or in other healthcare organizations, but they do not demand the same type of services as our regular roster of clinical and support staff or consumer library users. Because of the unique needs and usage patterns exhibited by med students, clerks and residents who access the Royal Columbian Hospital $(\mathrm{RCH})$ library, we conducted a needs assessment to help better serve these users. Methods: A quantitative and 
qualitative survey was designed to further investigate library usage and perception, with a focus on missing and overdue items. The survey was made available in print and electronic form to both medical students and residents as they began their placements at $\mathrm{RCH}$. Draw prizes of Toronto Notes Clinical Handbooks were offered as incentives to complete the survey. Results: The preliminary results of the survey indicate that a predominant amount of med students, clerks and residents visit medical libraries to use the computer/internet (97\%), or to use the photocopier/fax/printer $(86 \%) .42 \%$ of respondents indicated that forgetfulness was the primary reason for keeping library items past their due date; $76 \%$ indicated that overdue notices were preferred via email, and $71 \%$ that multiple and/or electronic copies of popular titles was preferred as the measure for reducing the incidence of missing and overdue items. Discussion (conclusions): This survey further emphasizes the need to engage library users in order to understand their perceptions and reasons for how they use library services. This knowledge, in turn, will help library staff to better meet their needs within the confines of a prescribed health care organization and budget.

\title{
EIDM (evidence-informed decision making) for the masses: lessons learned in a new rapid review process in public health
}

\author{
Diana Robertson, Mississauga, Rebecca Strange, and Jessie McGowan \\ Region of Peel, 7120 Hurontario St., PO Box 667, RPO Streetsville, ON; Region of Peel, 7120 Hurontario St., \\ PO Box 667, RPO Streetsville Mississauga, ON; 44 Evelyn Ave, Toronto, ON
}

Introduction: Peel Public Health has revolutionized the way it delivers library services in the past 3 years. The change has come about in answer to the need to provide a high-level of library services to support evidence-informed decision making (EIDM). Methods: The staff and organization of the library have changed to reflect the evidencebased model. Currently, two masters level librarians work in the library. The new focus is on the provision of evidence-based library services, in particular, the specialized support of rapid reviews (RRs). New processes have been developed to support RRs, which are being produced in-house. Data was collected on completed RR literature searches from Jan 2010 to November 2011. A searching protocol was developed to support RRs which uses preappraised literature based on the $6 \mathrm{~S}$ model. ${ }^{1,2}$ The goal of the protocol is to provide a framework for the searches and transparency of the process. Results: A total of 15 RRs were completed $(n=15)$. The goal of the literature search is to find 3 to 5 pre-appraised sources of evidence for each RR. The average number of articles found per search for each RR was 3.6. The average time spent searching was 4.6 hours. The majority of citations included in the RR were identified by a MEDLINE search $(79.6 \%)$, with the remainder being identified by other searches, including grey literature, other databases and hand searching. Discussion and conclusions: The searching protocol is an efficient tool for the Peel Public Health librarians to perform literature searches to support the RR process. The protocol is iterative and is being continually revised. The process will be presented and discussed, including the specific resources used.

\section{References}

1. DiCenso A, Bayley L, Haynes RB. ACP Journal Club. Editorial: Accessing preappraised evidence: fine-tuning the 5S model into a $6 \mathrm{~S}$ model. Annals of Internal Medicine 2009;151(6):JC3-2, JC3-3.

2. Robeson P, Dobbins M, DeCorby K, Tirilis D. Facilitating access to pre-processed research evidence in public health. BMC Public Health 2010;10:95. 


\title{
Methodology for examining the efficacy of screen-capture tutorials: a pilot study
}

\author{
Catherine Boden, Christine Neilson, and Jennifer Seaton \\ University of Saskatchewan Health Sciences Library; SHIRP, University of Saskatchewan; University of \\ Saskatchewan
}

Goal: This pilot study evaluated a research methodology for examining the efficacy of screen capture tutorials in teaching database search skills. Participants: Nine mental health practitioners from various practice settings and levels of experience with database searching participated in the study. Participants were randomly assigned to the experimental $(n=5)$ or control group $(n=4)$. Method: Each study session was conducted in the participants' workspace using the researchers' equipment. First, the participants' stage in the information search process and their existing mental model of the topic were assessed using a questionnaire (Spink and Dee, 2007) and concept mapping. Participants then searched PsycInfo for articles on a research topic of their choice, watched a 10-minute screen cast tutorial (experimental video demonstrated search tactics; control video described the Saskatchewan Health Information Resources Partnership), then re-conducted the search. Screen activity during searches was recorded using Morae software. Finally, participants completed a short structured interview, which was recorded on an iPad using Dictamus. Results: The Morae software was effective for recording, coding, and exporting search variables for analysis and provided rich information about participants' searches. Dictamus produced good quality recordings, which were easy to export from the iPad for transcription. Internet access was frequently problematic because of workplace security concerns. Discussion: The combination of concept maps, information stage questionnaire, and Morae recording provided rich information about health practitioners' research questions and search strategies. This method can prove useful for evaluating the efficacy and development of screen cast tutorials.

\section{Deciding to pursue answers to clinical questions: an exploratory study of stroke clinicians}

\author{
Lorie A. Kloda and Joan C. Bartlett \\ PhD candidate, McGill University, School of Information Studies, 3661 Peel Street, Montreal QC H3A 1X1
}

Introduction: In evidence-based practice, formulating a clinical question is the first step in a process that is intended to lead to effective information seeking. Research has demonstrated that clinicians, however, do not pursue answers to some of their questions. This study explored the information needs of occupational therapists, physiotherapists and speech-language pathologists working with patients with stroke and investigated how clinicians decide whether or not to pursue answers to their clinical questions. Methods: Through purposive, maximum variation sampling, 15 stroke clinicians with a range of 1 to 35 years experience were recruited into the study. Clinicians recorded their clinical questions in a journal over several weeks; a follow-up interview elicited more details about the questions recorded in the journal. Using thematic analysis, constraints and enablers to pursuing and answering clinical questions were identified. Findings: Decisions to pursue answers to clinical questions are complicated and multifaceted. Emerging themes include intrinsic reasons such as the clinician's belief in the existence of an answer and perceived self-efficacy. Extrinsic reasons include the perception of organizational support and workload demands. Clinicians' abilities to answer clinical questions are mediated by their awareness of sources and strategies for managing these sources in the context of their practice. Discussion: While information literacy skills and the availability of a librarian are important, many of the emergent themes suggest that the decision to pursue answers to clinical questions is far more complicated. Implications for health sciences librarianship will be discussed. 


\title{
Measuring the change in core practice-driving medical knowledge
}

\author{
Brian S. Alper \\ Editor-in-Chief, DynaMed, 10 Estes Street, Ipswich, MA 01938
}

Introduction: Medical knowledge is estimated to double every 2 to 19 years, most often estimated at 7 to 10 years. $16 \%$ of highly cited original research is subsequently contradicted. However, we do not know how often medical knowledge changes in areas thath affect clinical decisionmaking in daily practice. We will sample evidence syntheses guiding diagnosis and treatment for clinically important topics to determine how much the content has changed in the prior year, and how many of those changes reflect new evidence or guidelines that can change practice. Methods: 200 DynaMed topics representing common or serious conditions will be evaluated. Overview sections (syntheses of the best evidence and guidance informing clinical action) will be analyzed and compared to a date about 1-2 years prior. We will analyze the number of words, lines, concepts and evidence grades that have changed. We will classify each change as resulting from new evidence or guidance (the primary outcome for this analysis) or from user feedback or quality improvement. Results: Results are expected by June 2012. Discussion: This will provide the first representation of how frequently the core content guiding clinical practice is changed. In an era of evidence-based medicine and electronic data management using textbooks as a marker of standard practice is no longer warranted. Quantifying the frequency of change affecting core clinical practice has significant implications for the frequency of updating evidence-based clinical references, guidelines, medical education, and medical informatics applications.

\section{Using grounded theory to develop a model of information-seeking behaviour of senior nursing students}

\author{
Vicky Duncan \\ Health Sciences Library, University of Saskatchewan, 107 Wiggins Road, Saskatoon, SK S7K 5E5
}

Introduction: Nursing student education requires a strong emphasis on "evidence-based" knowledge, requiring advanced information seeking skills. Yet a number of studies reveal that nursing students, as well as practicing nurses, are not confident in their library research skills. This interdisciplinary, grounded theory study examined strategies used by students to locate resources and information for a class assignment, and identified barriers to their success. Methods: Purposive sampling was used to recruit 11 participants. Students were asked to journal their researching process while completing a class assignment; and semi-structured, open-ended, audiotaped interviews took place to discuss the students' journals and solicit additional data. Patterns of information seeking, strategies used to find information, and barriers to researching were identified. Results: Students' main concern was frustration caused by the challenge of choosing of words or phrases to query databases or resources. The basic social process was "discovering vocabulary", which was comprised of four subprocesses: confirming principles, testing the waters, selecting search terms, and adjusting search strategy. Results were compared to previous studies done with nursing students and clinical nurses. Discussion: Identifying students' main concern, basic social process and subprocesses using grounded theory methodology were key in the development of an information-seeking model for senior nursing students. The study also highlighted the importance of teaching nursing students searching skills, especially their primary database, CINAHL. Health sciences librarians can assist students by helping them identify strategies of identifying alternate vocabulary when querying information resources. 


\title{
Optimizing an informatics curriculum: using CanMEDS and ACRL standards to review our instruction
}

\author{
Mindy Thuna and Elena Springall \\ Science Liaison Librarian, HMALC, Library, 3359 Mississauga Road North, Mississauga, ON, L5L 1C6; Acting \\ Coordinator of Resource Sharing, Gerstein Science Information Centre, University of Toronto Libraries, 9 King's \\ College Circle, Toronto, ON, M5S 1 A5
}

Introduction: The CanMeds Framework, produced by the Royal College of Physicians and Surgeons of Canada, lists the competencies they feel are essential in medical education and practice and are a guiding document for medical education across Canada. Our objective in this study was to ensure that the informatics curriculum at our university addresses all the required competencies for a four-year undergraduate medical program and, if it did not, to determine the most efficient way to address these gaps. Method: A committee of academic and teaching hospital librarians identified the need for a curriculum review of our undergraduate medical offerings. A working group was formed to identify CanMeds competencies relevant to information literacy instruction, using the ACRL standards as a reference point. With the assistance of pertinent faculty of medicine instructors, these competencies were mapped to the existing curriculum, missing elements were identified, and strategies for ensuring the comprehensiveness of the curriculum were discussed. Results: The main results were the removal of a redundant class, restructuring of current offerings, and the addition of three new interventions. Discussion: This curriculum review identified that there were required competencies that were not being addressed. Our curriculum review drastically reshaped the way we provided informatics instruction at the time, and opened the door for ongoing discussions. A comprehensive informatics curriculum needs not only attention to required competencies, but open and ongoing dialogue between librarians, faculty, and students. 